\title{
A reliable murine model of disseminated infection induced by Talaromyces marneffei
}

\section{Juan He ( $\nabla$ hejuan@kmmu.edu.cn )}

Department of Dermatology and Venereology, First Affiliated Hospital of Kunming Medical University

Jia-Sheng Li

College of Pharmaceutical Sciences, Yunnan University of Traditional Chinese Medicine

\section{Yi-Qun Kuang}

NHC Key Laboratory of Drug Addiction Medicine, First Affiliated Hospital of Kunming Medicine University

Jun Li

College of Pharmaceutical Sciences, Yunnan University of Traditional Chinese Medicine Hong-Bin Li

Department of Dermatology and Venereology, First Affiliated Hospital of Kunming Medical University Zhe Li

Department of Dermatology and Venereology, First Affiliated Hospital of Kunming Medical University Hui-Ling Zhou

Department of Dermatology and Venereology, First Affiliated Hospital of Kunming Medical University

\section{Rui-Rui Wang}

College of Pharmaceutical Sciences, Yunnan University of Traditional Chinese Medicine

\section{Yu-Ye Li}

Department of Dermatology and Venereology, First Affiliated Hospital of Kunming Medical University

\section{Research Article}

Keywords: Talaromyces marneffei, Animal mode, Murine, Talaromycosis

Posted Date: March 26th, 2021

DOl: https://doi.org/10.21203/rs.3.rs-344933/v1

License: (a) (i) This work is licensed under a Creative Commons Attribution 4.0 International License. Read Full License

Version of Record: A version of this preprint was published at Mycopathologia on November 7th, 2021. See the published version at https://doi.org/10.1007/s11046-021-00596-4. 


\section{Abstract}

Talaromycosis (penicilliosis) caused by Talaromyces marneffei is one of the most important opportunistic infection diseases in tropical countries of South and Southeast Asia. Most infections occurred in individuals with human immunodeficiency virus (HIV) and the primarily reason for the increase in the number of the cases is HIV pandemic. The pathogenesis of T. marneffei infection is unclear. There is still no ideal animal model for studying Talaromycosis. In this study, we developed a stable, safe and maneuverable murine model that mimics human T. marneffei disseminated infection using T. marneffei yeast intraperitoneal injected to BALB/c nude mice. We successfully observed symptoms similar to those seen in clinical patients in this murine model, including skin lesions, hepatosplenomegaly, pulmonary infection and mesenteric lesions. We further studied the pathological changes of various tissues and organs in the infected animals to help better understand the severity of the infection. This model may provide a good tool for studying disseminated infection induced by $T$. marneffei.

\section{Introduction}

Talaromyces marneffei (T. marneffei), formerly named Penicillium marneffei [1], is a thermally dimorphic fungus that causes lethal mycosis called Talaromycosis (penicilliosis) in patients infected with human immunodeficiency virus (HIV) [2]. T. marneffei is the third most common opportunistic infection in HIVinfected patients in certain parts of Southeast Asia countries including Thailand, Vietnam and Southern China following extrapulmonary tuberculosis and cryptococcosis or pneumocystis [3]. T. marneffei can cause local infections and fatal disseminated infections. Disseminated infections were diagnosed in $86 \%$ of cases reported in the literature [4]. The mortality rate of patients with disseminated T. marneffei infection range from $10 \%-30 \%$, and even up to $97 \%$ if therapy is delayed [5-8]. As both basic and clinical knowledge about disseminated Talaromycosis is limited, laboratory models of the disease are needed.

Murine models have predominated for most investigators over the years regarding that mice and humans have similarities in organ systems, biochemistries, pathologies[9]. Several murine models have been developed to study Talaromycosis. Injection of $T$. marneffei yeast cells suspension into the lateral tail vein of mice had been used to assess the virulence of different strains [10]. A pulmonary infection murine model was created by intratracheal instillation of T. marneffei [11], whereas Liu et al infected mice by using a nebulizer to deliver T. marneffei conidia to the lungs of BALB/c nude mice housed in exposure chamber [12]. Though these studies provided some new insights into the pathogenesis of Talaromycosis, the methodologies are time-consuming or needing special equipment. A easy to operate animal model that can precisely mimic human T. marneffei infection is critical for study this disease and for drug screening and evaluation.

\section{Materials And Methods}

\section{Animals and ethics statement}


Congenitally athymic female BALB/c nude mice, weighing 18-21g, were purchased from the experiment animal center of the Kunming Medical University and were used in this experiment at the age of 6-8 weeks. The experimental protocol was approved by the Ethics Review Committee for Animal Experimentation of the Kunming Medical University. All mice were housed in a pathogen-free environment and received sterilized food and water at the Laboratory Animal Centre for Biomedical Sciences of the Kunming Medical University.

\section{Microorganisms and culture conditions}

T. marneffei strain ATCC18224 was purchased from American Type Culture Collection. The mycelial colony was cultured in brain heart infusion (BHI) plates incubated at $37^{\circ} \mathrm{C}$ for $72 \mathrm{~h}$ following repeated subculturing until the yeast cells appeared. The newly grown yeast colonies were transferred to an agitated flask (150 r.p.m) containing BHI broth and incubated at $37^{\circ} \mathrm{C}$ for $72 \mathrm{~h}$. Yeast were harvested by centrifuging at $3000 \mathrm{~g}$ for $10 \mathrm{~min}$, the resulting fungal suspensions were adjusted to the required concentrations using a hemocytometer.

\section{The infection process}

Ten mice received solution from $0.1 \mathrm{ml}$ of a suspension containing $5 \times 10^{7}$ yeast $/ \mathrm{ml}$. Ten mice received $0.1 \mathrm{ml}$ PBS as control. Twenty mice were used for survival assay. The rest were dissected and sampled for further study.

\section{Histopathological examination}

Another two mice, one mouse received solution from $0.1 \mathrm{ml}$ of a suspension containing $5 \times 10^{7}$ yeast $/ \mathrm{ml}$, and the other received $0.1 \mathrm{ml}$ PBS as control. On day 18 , two mice were used for collection of organ samples after euthanasia. For histopathological assay, half of each organ was fixed in $4 \%$ neutral buffered formalin, processed, and embedded in paraffin. Tissue sections were stained with hematoxylin and eosin (H\&E) and Periodic Acid-Silver-Meth-enamine (PASM) stain.

\section{Statistical analysis}

Graphs were plotted by using GraphPad Prism 5 software (GraphPad Software, La Jolla, CA). Comparisons between groups were tested using independent samples t-test. Survival data were analyzed by means of log-rank comparisons of Kaplan-Meier survival curves.

\section{Results}




\section{Establishment of the T. marneffei infection mouse model}

The body weight of the infected mice was significantly reduced compared to that of the negative controls (independent samples t-test: $t=-13.463, P=0.000$ ). Along with the gradual reduction of body weight of $T$. marneffei injected group, we observed a gradual increase of body weight in the PBS injected group. On Day 19 after injection, the mice in the infected group lost $15.7 \%$ of their body weight compared with the body weight before injection; the average body weight of the infected group was about $74.9 \%$ of that of the control group (Figure 1A). All infected mice died within 25 days after infection (Figure 1B), while all the control animal were alive. The first death occurred on day 20 in the infected group, six mice died on day 23, and the remaining 3 mice died on day 21, 24, 25 each (Figure 1B).

\section{Gross lesions and autopsy changes in BALB/c nude mice after infection with $T$. marneffei}

Mice in T. marneffei infected group developed skin lesions with central pitted papules (Figure 2 A-C), very similar to those in T. marneffei infected patients (Figure 2I). In particular, diffuse scattered nodular lesions were observed on the back of infected mice(Figure $2 \mathrm{D}$ ), which were very similar to the lesions on the back of T. marneffei infected patients(Figure $2 \mathrm{~J}$ ). Autopsy examination of the infected mice showed hepatomegaly (Figure 2E), splenomegaly (Figure 2F), lung injury (Figure 2G) and mesenteric nodules (Figure $2 \mathrm{H}$ ), and the fungus culture with mesenteric nodule showed T. marneffei (Supplementary Figure 1).

\section{Histopathological findings}

The damage of each organ and the yeast cells were further analyzed by H\&E and PASM staining respectively (Figure 3). Tissue section of the lung showing diffuse thickening of interalveolar septa, destruction of the alveolar and tracheobronchial structure, blood exudation, infiltration of inflammatory cells consisting mainly of leukocyte and mononuclear (Figure 3A-1), and macrophages and multinucleated giant cells loaded with large numbers of yeast cells (Figure 3A-2, green arrow). Tissue section of the skin showing damage to skin structure, partial tissue defect of epidermis and dermal, epidermis trochanterellus disappear at the site of fungal infection, structure boundary between dermis and epidermis is not clear, infiltration of inflammatory cells consisting mainly of leukocyte (Figure 3B-1), and fungal yeast was found in the epidermis, dermis and subcutaneous tissues (Figure 3B-2). The structure of the rectum is generally normal (Figure 3C-1), but a small amount of fungal yeast can be seen scattered in the mucosal layer (Figure 3C-2). Focal lesions are seen in the small intestine with deformed intestinal villus, infiltration of inflammatory cells, structures of serosal layer destroys and connects nodular lesions (Figure 3D-1), and fungal yeast was found in the lamina propria and nodular lesions (Figure 3D-2). Throughout the spleen there were extensive structural damages often with structure disappeared of white pulp, medullary substance and trabeculae lienis (Figure 3E-1) as well as numerous 
macrophages loaded with yeast cells (Figure 3E-2). Liver section primarily characterized by granuloma formation, leukocyte infiltration (Figure 3F-1) and macrophages containing many yeast-form cells of $T$. marneffei (Figure 3F-2). In the kidneys were characterized by scattered lesions (Figure. 3G-1) and yeast cells (Figure 3G-2) in the glomeruli and cortical interstitium. Fungal yeast is also found in the femoral bone marrow (Figure $3 \mathrm{H}-1$ ). No pathological changes and fungal yeast were observed in brain tissue and skeletal muscle tissue (Supplementary figure 2).

\section{Discussion}

In this study, we established a new experimental murine model of disseminated $T$. marneffei infection closely mimic the clinic feature of $T$. marneffei infection observed in humans. The most common clinical features observed in patients with $T$. mameffei include fever (98.0\%), anemia (74.8\%), and weight loss (71.6\%) [13]. These three symptoms are common non-specific symptoms of infectious diseases. Dissemination of the disease is characterized by skin lesions $(69.7 \%-85 \%)$, such as papules with central necrosis $[2,13]$. This characteristic lesion can be a single or first-episode symptom of Talaromycosis and has a strong suggestive role in the diagnosis of this disease [14]. It is therefore important to observe this particular lesion in a mouse model. The gross appearance of skin lesions in our model is consistent with that of human skin lesions in infected patients. Pulmonary infiltrates, lymphadenopathy, splenomegaly, and hepatomegaly were also noted in many patients [13]. Lesions in these organs were also found in the anatomy of our model group. Previous studies had reported diffuse infiltration of macrophages engorged with proliferating yeast cells in tissues[2,15] and a variety of organs including the skin, bone marrow, lymph nodes, liver, lung, bone, spleen, nasopharynx, bowel, kidney, pericardium, meninges [13]. We observed fungal yeasts in the skin, bone marrow, mesenteric nodules, liver, lung, spleen, kidney, rectum, small intestine tissues, but not in the brain and skeletal muscle. Except for the meninges, our model infection organs were almost identical to the infected organs of patients reported in the literature. Therefore, our murine model successfully mimicked deep-seated visceral fungal infection, and it was the first time that our murine model had skin lesions similar to those found in human patients.

Our modeling method has some advantages over the modeling method in the previous studies[10-12]). The first advantage is that it is easy to operate and no special equipment is needed. Recently, Liu et al had developed a murine pulmonary T. marneffei infection model, and they used BALB/c nude mice with a nebulizer to deliver $T$. marneffei conidia to the lungs with mice housed in exposure chamber[12]. The procedure needs a nebulizer driven by compressed air to generate a suspension containing conidia to aerosols and then input into a multi-animal exposure chamber. After passing through the exposure chamber the air is needed to filter before being sent into the exhaust system. The generated aerosol was connected to the exposure chamber, which was connected to an air filter though another tube to prevent spores from contaminating the environment. They first constructed a murine pulmonary model that would mimic human Talaromycosis utilizing inhalation exposure [12]. In their study, not only special experimental equipment but also air pollution and safety issues need to be considered. In another study, Sun et al injected T. marneffei yeast suspension the lateral tail vein of mice to establish the infection [10]. However, in operation, the fungal yeast easily agglomerates or precipitates, thus increasing the risk of 
pulmonary embolism in mice. Kudenken et al established T. marneffei infection in mice by intratracheal instillation, which is also a complex procedure and time-consuming [11]. The second advantage is that no big difference in the total survival time of each $T$. marneffei infected mouse in our model, with the first death on day 20 and all death on day 25. However, in the inhaled mouse model [12], animals began to die on day 15 after infection and some were still $35 \%$ alive on day 40 . In the tail vein injection mouse model [10], animals began to die on day 8 after infection and all the mice died on day 18 , animals of the intratracheal instillation mouse model began to die on day 33 after infection and all the mice died day 44 [11].

In conclusion, we successfully developed a stable, safe and maneuverable murine model of $T$. marneffei infection. The symptoms and diseased organs of this model are highly consistent with the disseminated T. marneffei infections in patients. This murine model may be used for understanding the pathogenesis of this disease.

\section{Declarations}

Author Contributions Juan He designed the study and prepared the manuscript; Jia-Sheng Li and Jun Li build animal model; Yi-Qun Kuang and Hong-Bin Li revised the prose; Zhe Li and Hui-Ling Zhou feed experimental animals; Rui-Rui Wang provided a laboratory site $\bigotimes Y u-Y e$ Li provided financial support.

Funding This research was funded by grants from Project for AIDS Bureau of Yunnan Province; "Tenthousand Talents Program" of Yunnan Province; Innovation Team of Department of Science and Technology of Yunnan Province (2018HC005); Natural Science Foundation of China (81560325, 81660737); the Joint Special Fund of Science and Technology Department of Yunnan Province-Yunnan University of Chinese Medicine (2017FF117-003)

\section{Compliance with Ethical Standards}

Conflict of interest The authors declare that they have no conflict of interest.

Consent to publish The authors affirm that human research participants provided informed consent for publication of the images in Figure 2l, 2J. Patients signed informed consent regarding publishing their data and photographs.

\section{References}

1. Samson RA, Yilmaz N, Houbraken J et al. Phylogeny and nomenclature of the genus Talaromyces and taxa accommodated in Penicillium subgenus Biverticillium. Studies in mycology 2011; 70: 159183 DOI: $10.3114 / \operatorname{sim} .2011 .70 .04$

2. Vanittanakom N, Cooper CR, Jr., Fisher MC et al. Penicillium marneffei infection and recent advances in the epidemiology and molecular biology aspects. Clinical microbiology reviews 2006; 19: 95-110 DOI: $10.1128 / \mathrm{cmr} \cdot 19.1 .95-110.2006$ 
3. Limper AH, Adenis A, Le T et al. Fungal infections in HIV/AIDS. The Lancet Infectious diseases 2017; 17: e334-e343 DOI: 10.1016/s1473-3099(17)30303-1

4. Nittayananta W. Penicilliosis marneffei: another AIDS defining illness in Southeast Asia. Oral diseases 1999; 5: 286-293 DOI: 10.1111/j.1601-0825.1999.tb00091.x

5. Jiang J, Meng S, Huang S et al. Effects of Talaromyces marneffei infection on mortality of HIV/AIDS patients in southern China: a retrospective cohort study. Clinical microbiology and infection : the official publication of the European Society of Clinical Microbiology and Infectious Diseases 2019; 25: 233-241 DOI: 10.1016/j.cmi.2018.04.018

6. Hu Y, Zhang J, Li X et al. Penicillium marneffei infection: an emerging disease in mainland China. Mycopathologia 2013; 175: 57-67 DOI: 10.1007/s11046-012-9577-0

7. Chariyalertsak S, Supparatpinyo K, Sirisanthana T et al. A controlled trial of itraconazole as primary prophylaxis for systemic fungal infections in patients with advanced human immunodeficiency virus infection in Thailand. Clinical infectious diseases : an official publication of the Infectious Diseases Society of America 2002; 34: 277-284 DOI: 10.1086/338154

8. Son VT, Khue PM, Strobel M. Penicilliosis and AIDS in Haiphong, Vietnam: evolution and predictive factors of death. Medecine et maladies infectieuses 2014; 44: 495-501 DOI: 10.1016/j.medmal.2014.09.008

9. Waterston RH, Lindblad-Toh K, Birney E et al. Initial sequencing and comparative analysis of the mouse genome. Nature 2002; 420: 520-562 DOI: 10.1038/nature01262

10. Sun J, Li X, Feng $P$ et al. RNAi-mediated silencing of fungal acuD gene attenuates the virulence of Penicillium marneffei. Medical mycology 2014; 52: 167-178 DOI: 10.1093/mmy/myt006

11. Kudeken N, Kawakami K, Kusano $\mathrm{N}$ et al. Cell-mediated immunity in host resistance against infection caused by Penicillium marneffei. Journal of medical and veterinary mycology : bi-monthly publication of the International Society for Human and Animal Mycology 1996; 34: 371-378 DOI: $10.1080 / 02681219680000671$

12. Liu $Y$, Huang $X, Y i X$ et al. Detection of Talaromyces marneffei from Fresh Tissue of an Inhalational Murine Pulmonary Model Using Nested PCR. PloS one 2016; 11: e0149634 DOI: 10.1371/journal.pone.0149634

13. Duong TA. Infection due to Penicillium marneffei, an emerging pathogen: review of 155 reported cases. Clinical infectious diseases : an official publication of the Infectious Diseases Society of America 1996; 23: 125-130 DOI: 10.1093/clinids/23.1.125

14. Cao C, Xi L, Chaturvedi V. Talaromycosis (Penicilliosis) Due to Talaromyces (Penicillium) marneffei: Insights into the Clinical Trends of a Major Fungal Disease 60 Years After the Discovery of the Pathogen. 2019; 184: 709-720 DOI: 10.1007/s11046-019-00410-2

15. Chan YF, Chow TC. Ultrastructural observations on Penicillium marneffei in natural human infection. Ultrastructural pathology 1990; 14: 439-452 DOI: 10.3109/01913129009007223

\section{Figures}



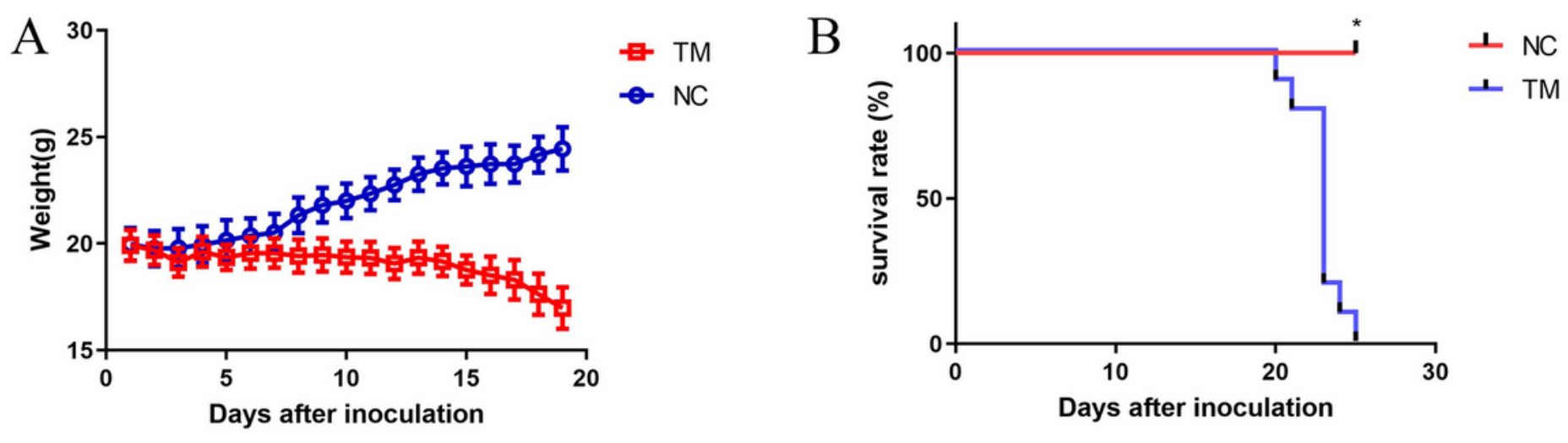

Figure 1

Comparison of Body weights (A) and the survival rate (B) between BALB/C nude mice infected with $T$. marneffei and mice inoculated with PBS TM group inoculated with T. marneffei. Control animals were inoculated with PBS. All mice were monitored daily for body weight until there were dead mice. Each point shows mean and SD (TM group, $n=10$; control group, $n=10$. *: $P<0.001$.)

Anatomical manifestations of T. marneffei infected mice and Skin lesions of patients
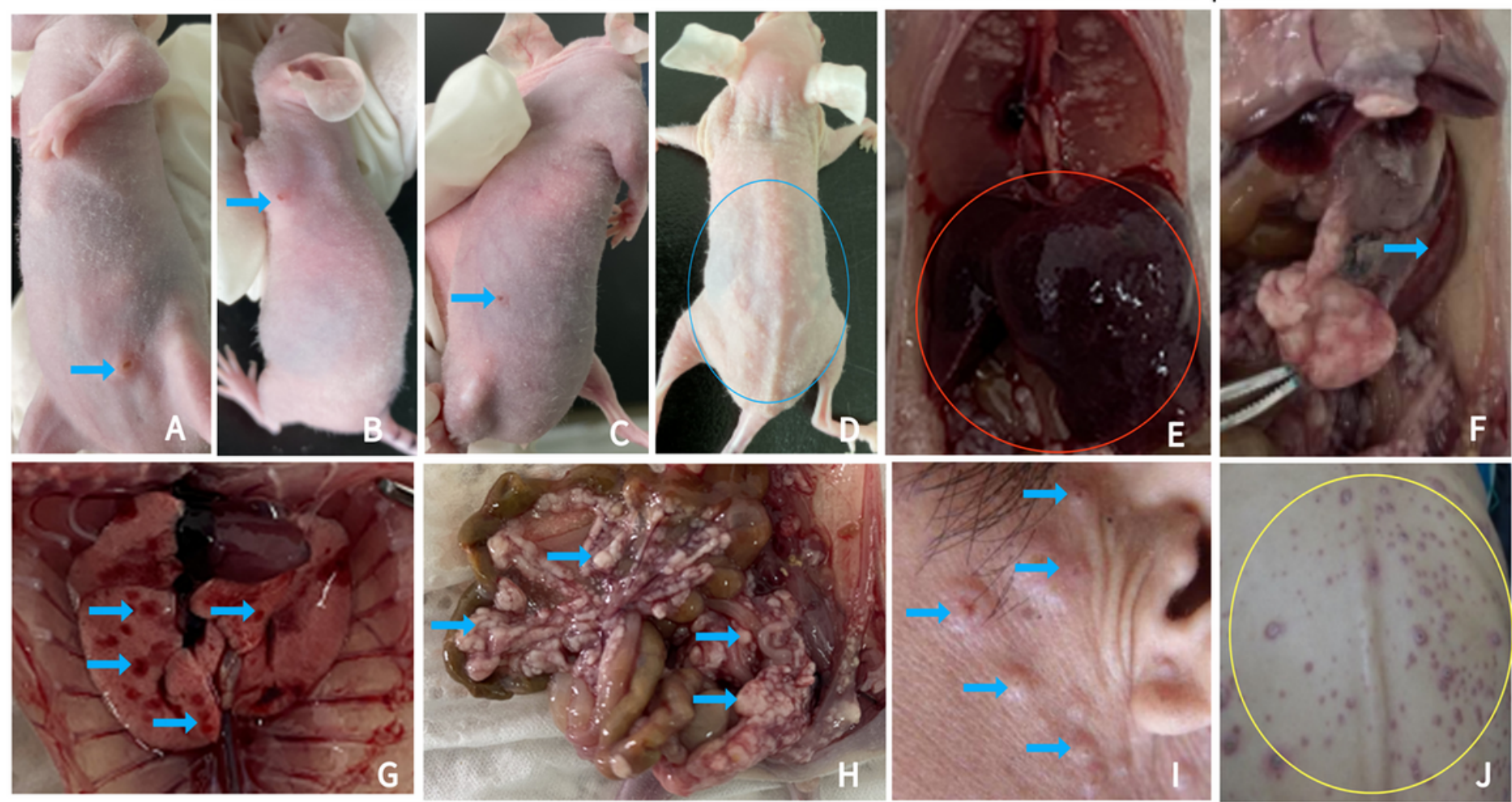

\section{Figure 2}

Anatomical manifestations of T. marneffei infected mice and corresponding skin lesios picture of T. marneffei infected patients A-C: Skin lesions in different parts of BALB/c nude mice infected with $T$. marneffei. D:Nodular lesion of BALB/c nude mice infected with T. marneffei. E: Hepatomegaly of BALB/C nude mice infected with T. marneffei. F: Splenomegaly of BALB/c nude mice infected with T. marneffei. 
G:Pulmonary lesions of BALB/c nude mice infected with T. marneffei. $\mathrm{H}$ : Mesenteric lesion of BALB/C nude mice infected with T. marneffei. I: Facial skin lesions of patients with Talaromycosis. J: Back lesions in patients with Talaromycosis.
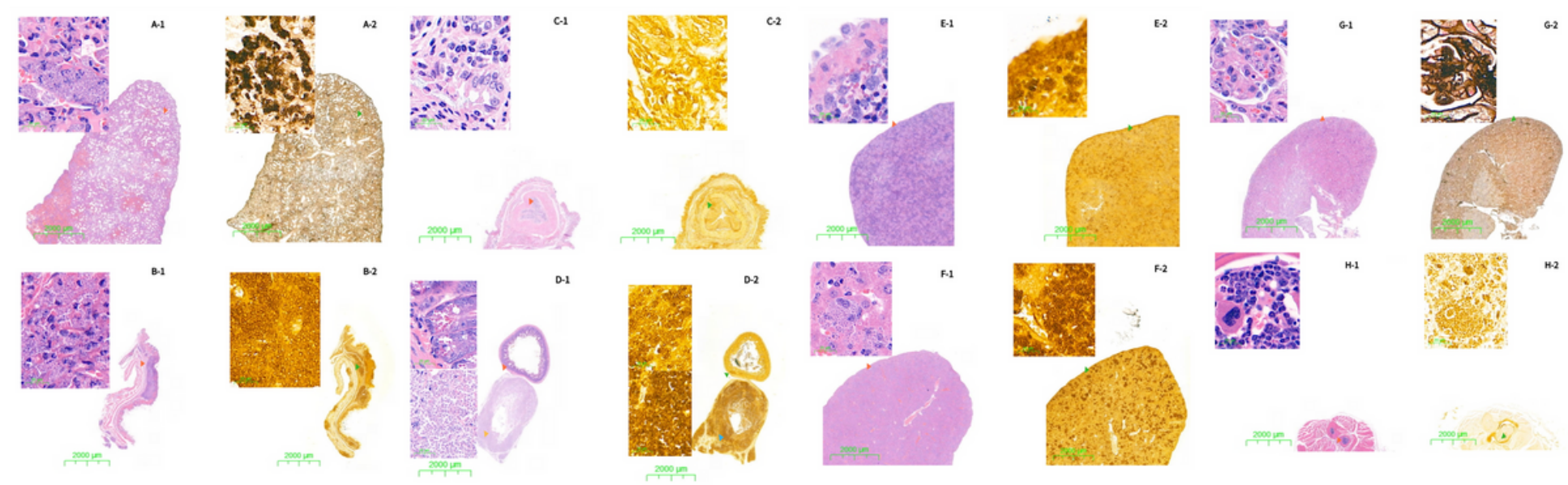

\section{Figure 3}

HE (n-1) and PASM (n-2) analyses of BALB/c nude mice infected with T. marneffei Fungal yeasts were observed in the lung $(A)$, shin $(B)$, rectum $(C)$, small intestine $(D)$, mesenteric nodules $(D)$, spleen (E), liver $(F)$, kidney $(G)$, bone marrow tissues $(H)$ of the infected mice. Magnification: The lower right corner $\times 4$, scale bars: $2000 \mu \mathrm{m}$. The top left corner $\times 400$, scale bars: $20 \mu \mathrm{m}$.

\section{Supplementary Files}

This is a list of supplementary files associated with this preprint. Click to download.

- Supplementary.docx 